\title{
FURTHER OBSERVATIONS OF THE STRUCTURE OF THE CHROMOSPHERE-CORONA TRANSITION REGION FROM LIMB AND DISK INTENSITIES
}

\author{
W. M. BURTON, C. JORDAN, A. RIDGELEY and R. WILSON* \\ SRC Astrophysics Research Division of the Radio and Space Research Station, \\ Culham Laboratory, Abingdon, Berkshire, England
}

\begin{abstract}
Further observations of limb and disk intensity ratios of emission lines in the EUV solar spectrum were obtained on a Skylark rocket flight on 5 August 1971. Analysis of the data has shown that the observations support the existence of a steep rise in temperature in the transition region between $T_{e} \sim 6 \times 10^{4} \mathrm{~K}$ and $3 \times 10^{5} \mathrm{~K}$. The average absolute height of the transition region above the visible limb has been measured with a greater accuracy than previously possible and is $1700 \mathrm{~km} \pm 700 \mathrm{~km}$. An independent method using arc-length measurements of spectrum lines gives an absolute height of $2100 \mathrm{~km} \pm 850 \mathrm{~km}$. Emission from lines which are optically thick in spicules is observed to extend to heights of $10000 \mathrm{~km}$ above the transition region. The observed decrease of FeII emission with height is consistent with current spicule statistics.

The limb to disk intensity ratios of pairs of lines from a given ion, one of which lies at a wavelength shorter than the head of the Lyman continuum and the other at a wavelength longer than the head of the Lyman continuum, have been studied, and the observed absorption at the limb by the Lyman continuum can be explained by spicular material with $N_{\mathrm{e}}=1.4 \times 10^{11} \mathrm{~cm}^{-3}$ and $T_{\mathrm{e}}=1.1 \times 10^{4} \mathrm{~K}$.

The abundance of iron has been derived from the chromosphere emission lines of Fe II. This value agrees, within the experimental accuracy of a factor of two, with the value of $N(\mathrm{Fe}) / N(\mathrm{H})=4 \times 10^{-5}$ found from previous analyses of photospheric and coronal lines.
\end{abstract}

\section{Reference}

Burton, W. M., Jordan, C., Ridgeley, A., and Wilson, R.: 1973, Astron. Astrophys. $27,101$.

\section{DISCUSSION}

After C. Jordan's paper, a lengthy discussion took place involving mostly the point of knowing whether one can assume the chromosphere below $1700 \mathrm{~km}$ to be homogeneous or not, and what forces are commanding the spicules to be in equilibrium - more or less - with the interspicular medium above $1700 \mathrm{~km}$.

Sturrock raised the two following points:

Sturrock: I would like to make two comments:

(1) The thickness of most of the region around the spicule depends very much upon what you may assume on the conduction coefficient. If you have a model of the spicule's cool gas moving along the magnetic field the heat conduction is transverse to the magnetic field, and the thickness of the spicular transition region would be very much less than one would otherwise expect.

* Dept. of Physics and Astronomy, University College London. 
(2) The models which you (C. Jordan and J. T. Jefferies) have drawn show spicules going down to the photosphere. Of course gas is being shot up, so some force is moving the gas up. This may mean that there is a bottom to the spicule, so that it is like a bullet of gas being pulled up. I think there are two possible forces which can move the gas upwards. One is the magnetic stress, and the other one is gas pressure. If the latter is true, it means that below the cool gas in the spicule there is some very hot gas pushing it up. This is possible if there is a mechanism for converting magnetic energy into heat. It might possibly raise the temperature, locally, to $10^{6} \mathrm{~K}$ and the pressure increase then shoots up the mass of cool gas like a bullet. If this is the case then there will be a thin transition region on the underside of this mass of gas which one must incorporate into the models.

Jordan: We can't say anything about the spicules below $1700 \mathrm{~km}$.

Thomas noted that the Jordan description fits the rather old model derived from the analysis of the $\mathrm{H}$ continuum. He commented that there must be heating near the bottom of the transition region, where $T_{\mathrm{e}}$ rises quickly from 9000 to $20000^{\circ}$. Such behavior must hold also across the spicules. What fixes the temperature variation across the spicule is the heating mechanism, and the behavior of the Lyman continuum opacity.

Schmidt: I maintain my statement that only magnetic stresses really can provide the necessary forces, not the pressure gradients.

Meyer: I just want to ask a question about the transition zone at the surrounding of the spicule. I have the impression that what Dr Thomas said was that you would have there the normal transition zone and you would also have the interspicular region, whereas in the remarks that Dr Schmidt made yesterday and which Dr Sturrock just brought up again, would indicate that the transition zone around the mantle of the spicule should be very small and it would be hard to believe that there could be any contribution of that to the interspicular medium. Would you agree with that statement?

Jordan: I don't mind how thin it is around the spicule, there is no inconsistency with having it any thinner.

Thomas: Be careful what you are talking about; you are not talking in terms of geometry, you are talking in terms of opacity! So the need here is to balance whatever energy input there is against the behavior of temperature and the ground-state population of hydrogen, i.e., against the rate of fall-off of Lyman opacity. But I have to couple the opacity with temperature and all I say is that it is not going to be uniform across the whole spicule.

Brueckner: In a paper (which was just published two months ago) we assumed a very similar model and tried to fit the eclipse observations into a transition zone like the one assumed by Jordan. The statistics of Beckers were used to derive the transition zone intensities as function of height as observed from the eclipse. Now we agree that a large part of the emission has to come from these spicules - the question is open whether the layer under the spicule is a very thin layer with very high temperature gradients, and we have here one point where we do not agree with Carole Jordan's results. At this altitude of approximately $1700 \mathrm{~km}$, we would observe, during the eclipse, a jump in the integrated intensity when the Moon moves over the $1700 \mathrm{~km}$ altitude lines; we have tried everything that we could to find this jump and we cannot find it! It is a very smooth curve, the intensity of these transition zone lines goes very smoothly out - there is no jump at $1700 \mathrm{~km}$....

The discussion ended there - more or less - on a disagreement on the eclipse interpretation; Thomas noted that the second derivative of the eclipse curves is involved, which makes Brueckner's point apparently rather weak. Brueckner then stated that ATM observations might solve the apparent dilemma of the abrupt (or not) change of character of the photosphere above $1700 \mathrm{~km}$. 